Pacific Journal of Mathematics

EXISTENCE OF STRONG SOLUTIONS TO SINGULAR 


\title{
EXISTENCE OF STRONG SOLUTIONS TO SINGULAR NONLINEAR EVOLUTION EQUATIONS
}

\author{
M. A. FREEDMAN
}

The nonlinear evolution equation $u^{\prime}(t)+A(t) u(t) \ni 0$ is studied under conditions which permit $A(t)$ to be singular at $t=0$. Application is then made to examples of partial differential equations having time dependent coefficients which blow up at the origin.

1. Introduction. We are concerned with gaining basic insights into existence of strong solution to the abstract Cauchy problem $(\mathrm{ACP})_{s}$

$$
\begin{gathered}
\frac{d u}{d t}+A(t) u(t) \ni 0, \quad 0 \leq s \leq t \leq T, \\
u(s)=x,
\end{gathered}
$$

when hypotheses are imposed on $A(t)$ which are weak enough to allow for singularity at $t=0$. Here $u:[s, T] \rightarrow X$, where $X$ is an arbitrary Banach space. The operators $\{A(t)\}_{s \leq t \leq T}$ are assumed to satisfy

(A.0) For a.e. $t$ in $[0, T], A(t)$ is a nonlinear, possibly multivalued operator on $X$,

(A.1) There exists $\bar{D} \subseteq X$ such that $\overline{\text { Dom } A(t)} \equiv \bar{D}$ for a.e. $t$, and in addition, the $m$-accretive type conditions: for some real number $\omega$ and for $\lambda_{0}$ satisfying $\omega \lambda_{0}<1$,

(A.2) $\operatorname{Ran}(I+\lambda A(t)) \supseteq \bar{D}$ for a.e. $t$ and $0<\lambda<\lambda_{0}$,

(A.3) For a.e. $t$, the resolvent operator $J_{\lambda}(t) \equiv[I+\lambda A(t)]^{-1}$ exists as a Lipschitz mapping on $\bar{D}$ with $\left\langle J_{\lambda}(t)\right\rangle_{\text {Lip }} \leq(1-\omega \lambda)^{-1}$.

Now $u(t)$ shall be referred to as a strong solution to $(\mathrm{ACP})_{s}$ if either

(i) $u(t)$ is continuous on $[s, T]$ and $u(s)=x$,

(ii) $u(t)$ is differentiable almost everywhere and satisfies the differential equation of $(\mathrm{ACP})_{s}$ a.e.,

(iii) $u(t)$ is absolutely continuous on $[s, T]$

or

(i) and (ii) as above and

(iii) $^{\prime} \quad u(t)$ is absolutely continuous on compact subsets of $(s, T)$. 
The difference between (S.1) and (S.2) may be viewed as follows. Under (S.1), $u(t)$ satisfies

$$
u(t)=x+\int_{s}^{t} u^{\prime}(\xi) d \xi \quad \text { for all } t \in[s, T],
$$

while under (S.2), $u(t)$ satisfies the weaker property

$$
u(t)-u(p)=\int_{p}^{t} u^{\prime}(\xi) d \xi \quad \text { for all } p, t \in(s, T) .
$$

Both of these notions of strong solution appear in the literature; e.g. (S.1) is used in [7] and (S.2) appears in [2].

Suppose that (ACP) ${ }_{s}$ has a strong solution (S.1) for every $0<\mathrm{s}<T$ and every $x \in \bar{D}$. We ask, will (ACP) $)_{0}$ have strong solution (S.1) or (S.2) for all $x \in \bar{D}$ ? In [4], it is shown that $A(t)$ can be so singular at $t=0$ that the answer is in general no even when

(B.1) Each $A(t)$ is a bounded linear (single-valued) operator defined on all of $X$,

(B.2) (A.2) and (A.3) are satisfied with $\omega=0$ and $\bar{D}=X$,

(B.3) The resolvent operator $J_{\lambda}(t) x$ is a jointly continuous function of $(\lambda, t, x) \in[0, \infty) \times[0, T] \times X$.

Existence of generalized solution to $(\mathrm{ACP})_{s}$ as a product integral of resolvents

$$
u(t)=\prod_{s}^{t} J_{d \xi}(\xi) x, \quad 0 \leq s \leq t \leq T,
$$

is also studied in [4]. Here, $\Pi_{s}^{t} J_{d \xi}(\xi) x$ denotes the limit

$$
\lim _{n \rightarrow \infty} \prod_{i=q(s, n)}^{q(t, n)} J_{\tau_{i}^{n}-\tau_{i-1}^{n}}\left(\xi_{i}^{n}\right) x
$$

where:

(i) for each $n, P^{n}=\left\{\tau_{i}^{n}\right\}_{i=0}^{N_{n}}$ is some partition of [0,T]; i.e. $0=\tau_{0}^{n} \leq$ $\tau_{1}^{n}<\cdots<\tau_{N_{n}}^{n}=T$, and each $\xi_{i}^{n}$ is some point in $\left(\tau_{i-1}^{n}, \tau_{i}^{n}\right]$,

(ii) for each $n$ and any $\sigma \in(0, T), q(\sigma, n)$ is that index for which $\sigma \in\left(\tau_{q(\sigma, n)-1}^{n}, \tau_{q(\sigma, n)}^{n}\right]$

(iii) $\left\|P^{n}\right\|=\max _{1 \leq i \leq N_{n}}\left(\tau_{i}^{n}-\tau_{i-1}^{n}\right) \rightarrow 0$ as $n \rightarrow \infty$.

It was found, under (B.1)-(B.3) and existence of strong solution (S.1) or (S.2) to (ACP) $)_{s}$ for all $0<s<T$ and all $x \in X$, that in general there is no sequence of partitions $P^{n}$ with $\left\|P^{n}\right\| \rightarrow 0$ for which $(\mathrm{ACP})_{0}$ has a generalized (i.e. product integral) solution for all $x \in X$.

We are thus led to ask what minimum hypotheses beyond (A.0)-(A.3) would allow us to identify those $x \in X$ for which $(\mathrm{ACP})_{s}$ has solution-either strong or generalized-for all $s \in[0, T)$. In $\$ 2$ we present such hypotheses which are mild enough to allow for $A(t)$ to be 
singular at $t=0$ and $\S 3$ gives examples of partial differential equations which serve to illustrate these hypotheses. In $\S 4$ we argue that under hypotheses (B.1)-(B.3) alone, set even in a separable Hilbert space, $A(t)$ can be so badly behaved as to discourage hope for proving existence of solution to $(\mathrm{ACP})_{0}$.

2. Existence for $s=0$. Assuming (A.0)-(A.3), for a.e. $t$, let $A_{\lambda}(t)$ denote the single-valued Yosida approximations to $A(t)$ given by, for each $\lambda \in\left(0, \lambda_{0}\right)$,

$$
A_{\lambda}(t)=\frac{I-J_{\lambda}(t)}{\lambda}, \quad \operatorname{Dom}\left(A_{\lambda}(t)\right)=\bar{D}
$$

Now define for a.e. $t \in[0, T]$ and each $x \in \bar{D}$,

$$
|A(t) x| \equiv \sup _{0<\lambda<\lambda_{0}}\left\|A_{\lambda}(t) x\right\| .
$$

The notation $|A(t) x|$ is in accordance with [2, p. 61]. There it is shown that for fixed $x$ and $t,\left\|A_{\lambda}(t) x\right\|$ is a nonincreasing function of $\lambda>0$. Hence, for every $x \in \bar{D},|A(t) x|$ exists in $[0, \infty]$ as the monotone increasing limit of $\left\|A_{\lambda}(t) x\right\|$ as $\lambda \rightarrow 0^{+}$. The set

$$
\hat{\mathscr{D}}(t)=\{x \in \bar{D}:|A(t) x|<\infty\}
$$

is called the generalized domain of $A(t)$, for, if $x$ is in the domain of $A(t)$, one may show that $|A(t) x|<\infty$. Thus $\operatorname{Dom}(A(t)) \subseteq \hat{\mathscr{D}}(t)$; in general the inclusion is proper, though when $X$ is reflexive, $\operatorname{Dom}(A(t))=\hat{\mathscr{D}}(t)$.

Next, we introduce the conditions

(P.1) $\mathscr{D}(t) \equiv \hat{\mathscr{D}}$ is independent of $t$ for a.e. $t \in[0, T]$,

(P.2) For at least one $x \in \hat{\mathscr{D}}$ there exists $\varepsilon>0$ such that the functions $\left\{\left\|A_{\lambda}(\xi) x\right\|\right\}_{\lambda}$ belong to $L^{1}(0, \varepsilon)$ and satisfy

$$
\sup _{0<\lambda<\lambda_{0}} \int_{0}^{\varepsilon}\left\|A_{\lambda}(\xi) x\right\| d \xi<\infty .
$$

(P.1) and (P.2) were motivated by the Crandall-Pazy-Evans conditions [2], [3]:

(C.1) There exists a Bochner integrable function $h:[0, T] \rightarrow X$ and a nondecreasing continuous function $L$ such that

$$
\left\|A_{\lambda}(t) x-A_{\lambda}(p) x\right\| \leq\|h(t)-h(p)\| L(\|x\|)
$$

for $0<\lambda<\lambda_{0}$, a.e. $p, t \in[0, T]$ and $x \in \bar{D}$.

(C.2) There exists a measurable function $h:[0, T] \rightarrow X$ of bounded variation and $L$ as above such that

$$
\begin{aligned}
& \qquad\left\|A_{\lambda}(t) x-A_{\lambda}(p) x\right\| \leq\|h(t)-h(p)\| L(\|x\|)\left(1+\left\|A_{\lambda}(p) x\right\|\right) \\
& \text { for } 0<\lambda<\lambda_{0} \text {, a.e. } p, t \in[0, T] \text { and } x \in \bar{D} \text {. }
\end{aligned}
$$


Note that (P.1) and (P.2) are implied by either (C.1) or (C.2). Indeed, they may be viewed as "point-wise" generalizations to the uniform time dependence expressed in (C.1) and (C.2). In [3] it is proved that (A.0)-(A.3) and either (C.1) or (C.2) imply existence of the product integral $\Pi_{s}^{t} J_{d \xi}(\xi) x$ for some sequence of partitions $\left\{P^{n}\right\}$ of $[s, T]$ having $\left\|P^{n}\right\| \rightarrow 0$ as $n \rightarrow \infty$. Futhermore, when (ACP) ${ }_{s}$ has a strong solution (S.2) for some $x \in \bar{D}$, then this solution agrees with $\Pi_{s}^{t} J_{d \xi}(\xi) x$ for all $t \in[s, T]$.

In the counterexample of [4], besides (B.1)-(B.3), conditions (C.1) and (C.2) are both satisfied for a.e. $p$ and $t$ in any compact subinterval of $(0, T]$. Yet, for some $x_{0} \in X$, the product integral $\Pi_{0}^{t} J_{d \xi}(\xi) x_{0}$ was shown not to converge along any sequence of partitions $\left\{P^{n}\right\}$ of $[0, T]$ for which $\lim _{n \rightarrow \infty}\left\|P^{n}\right\|=0$, nor did (ACP) $)_{0}$ with $x=x_{0}$ have strong solution (S.1) or (S.2). Nonetheless, in this paper we have

TheOREM 2.1. Assume that $A(t)$ satisfies (A.0)-(A.3) and (P.1)-(P.2). Let $\mathscr{F}$ be the set of all elements of $\hat{\mathscr{D}}$ which satisfy the condition (P.2). Suppose for all $0<s<T,(\mathrm{ACP})_{s}$ has an (S.1) strong solution $u(t)$ for

(1) each $x \in \bar{D}$

or

(2) each $x \in \hat{\mathscr{D}}$; and $u(t) \in \hat{\mathscr{D}}$ for each $t$.

Then (ACP) ${ }_{0}$ has a strong solution (S.2) for each $x \in \overline{\mathscr{F}}$.

THEOREM 2.2. In addition to (A.0)-(A.3) and (P.1), (P.2), assume that for each $0<a<T$ there exist functions $h=h_{a}$ and $L=L_{a}$ for which $A$ satisfies either (C.1) or (C.2) for $0<\lambda<\lambda_{0}$, a.e. $p, t \in[a, T]$ and $x \in \bar{D}$. Then, for all $x \in \overline{\mathscr{F}}$, the improper product integral $\tilde{W}(t, 0) \equiv$ $\lim _{p \rightarrow 0+} W(t, p) x$ exists on $(0, T]$.

Furthermore, $\tilde{W}(t, 0) x$ is a generalized solution in the sense that if $u(t)$ is a strong solution (S.1) to $(\mathrm{ACP})_{0}$, then $u(t)=\tilde{W}(t, 0) x$.

Theorem 2.1 depends on the following proposition, the proof of which closely follows the proofs of Theorems 1.1 and 2.3 of [5].

Proposition 2.1. Let $A(t)$ be such that (A.0)-(A.3) hold and a strong solution (S.1) to (ACP) $)_{s}$ exists for all $0<s<T$ and $x \in \bar{D}$ and let $F$ be Bochner integrable on $[0, T]$. Given any sequence of partitions $P^{n}=\left\{\tau_{i}\right\}_{i=0}^{N_{n}}$ of $[0, T]$ satisfying

(M.1) There is a constant $B$ such that $\left\|P^{n}\right\| \leq B\left\{\min _{1 \leq i \leq N_{n}}\left(\tau_{i}^{n}-\tau_{i-1}^{n}\right)\right\}$, for all $n$,

(M.2) $\left\|P^{n}\right\| \rightarrow 0$ as $n \rightarrow \infty$, 
then there exist points $\xi_{k}^{n} \in\left(\tau_{k-1}^{n}, \tau_{k}^{n}\right]$ such that the limit (1.1) converges uniformly for $s \leq t \leq T$. Now define

$$
W(t, s) x \equiv \text { limit of }(1.1), \quad x \in \bar{D} .
$$

Then for all $0<r \leq s \leq t \leq T$ and $x \in \bar{D}$ we have $W(s, r) x \in \bar{D}$ and

$$
W(t, s) W(s, r) x=W(t, r) x .
$$

As well, if $u(t)$ is an (S.1) solution to $(\mathrm{ACP})_{s}$ with $u(s)=x \in \bar{D}$ and $v(t)$ is an (S.1) solution with $v(s)=y \in \bar{D}$ then for all $s \leq t \leq T$

$$
W(t, s) x=u(t)
$$

and

$$
\|v(t)-u(t)\| \leq e^{\omega(t-s)}\|x-y\| .
$$

Finally, we have

$$
\int_{s}^{t} F(\xi) d \xi=\lim _{n \rightarrow \infty} \sum_{k=q(s, n)}^{q(t, n)} F\left(\xi_{k}^{n}\right)\left(\tau_{k}^{n}-\tau_{k-1}^{n}\right) \quad \text { for all } 0 \leq s \leq t \leq T \text {. }
$$

From (2.3) we see that (S.1) strong solutions to (ACP) $)_{s}$ are unique. We introduce the notation $V(t, s) x$ to denote such a solution. Thus (2.2) becomes

$$
W(t, s) x=V(t, s) x
$$

LEMMA 2.1. Under the hypotheses of Theorem 1.1, if $x \in \mathscr{F}$ then there is $C>0$ and $\varepsilon>0$ such that

$$
\|V(t, s) x-V(t, r) x\| \leq C e^{\omega(t-s)} \int_{r}^{s}|A(\xi) x| d \xi
$$

for all $0 \leq r \leq s \leq \varepsilon, t \leq T$.

Proof. Given $x \in \mathscr{F}$ let $\varepsilon$ be as in condition (P.2), where we observe that (2.1) is equivalent (via the Monotone Convergence theorem) to integrability of $|A(\xi) x|$ on $[0, \varepsilon]$. Now, by (2.4), it will suffice to prove that

$$
\begin{aligned}
& \|W(t, s) x-W(t, r) x\| \\
& \quad \leq C e^{\omega(t-s)} \int_{r}^{s}|A(\xi) x| d \xi, \quad 0<r \leq s \leq \varepsilon, t \leq T .
\end{aligned}
$$

By Proposition 2.1 we have

$$
\begin{aligned}
\|W(t, s) x-W(t, r) x\| & =\|W(t, s) x-W(t, s) W(s, r) x\| \\
& \leq e^{\omega(t-s)}\|W(s, r) x-x\| .
\end{aligned}
$$

Hence it will suffice to prove that

$$
\|W(s, r) x-x\| \leq C \int_{r}^{s}|A(\xi) x| d \xi
$$


Let $P^{n}=\left\{\tau_{i}^{n}\right\}_{i=0}^{N_{n}}$ be a sequence of partitions of $[0, T]$ satisfying (M.1) and (M.2) and let $\xi_{k}^{n} \in\left(\tau_{k-1}^{n}, \tau_{k}^{n}\right]$ be as in Proposition 2.1. Then

$$
\begin{aligned}
& \left\|\prod_{i=q(r, n)}^{q(s, n)} J_{\tau_{i}^{n}-\tau_{i-1}^{n}}\left(\xi_{i}^{n}\right) x-x\right\| \\
& \quad=\left\|\sum_{i=q(r, n)}^{q(s, n)}\left(\prod_{j=i}^{q(s, n)} J_{\tau_{j}^{n}-\tau_{j-1}^{n}}\left(\xi_{j}^{n}\right) x-\prod_{j=i+1}^{q(s, n)} J_{\tau_{j}^{n}-\tau_{j-1}^{n}}\left(\xi_{j}^{n}\right) x\right)\right\| \\
& \quad \leq \sum_{i=q(r, n)}^{q(s, n)}\left[1-\left(\tau_{i}^{n}-\tau_{i-1}^{n}\right) \omega\right]^{-(q(s, n)-i+1)}\left|A\left(\xi_{k}^{n}\right) x\right|\left(\tau_{i}^{n}-\tau_{i-1}^{n}\right) \\
& \quad \leq \exp \left(2(q(s, n)-q(r, n))\left\|P^{n}\right\||\omega|\right) \sum_{i=q(r, n)}^{q(s, n)}\left|A\left(\xi_{k}^{n}\right) x\right|\left(\tau_{i}^{n}-\tau_{i-1}^{n}\right),
\end{aligned}
$$

where we have used the bound: $(1-\theta)^{-1} \leq e^{-\theta}, 0 \leq \theta \leq 1 / 2$. By (M.1), $(q(s, n)-q(r, n))\left\|P^{n}\right\| \leq B$. The lemma now follows with $C=e^{2 B|\omega|}$ upon letting $n \rightarrow \infty$.

Proof of Theorem 2.1. Assume case (1) holds, i.e. (ACP) ${ }_{s}$ has strong (S.1) solution $V(t, s) x$ for all $x \in \bar{D}$ and $0<s<T$. By Lemma 2.1 we may conclude that given $x \in \mathscr{F}$, for each $0<t \leq T$,

$$
w(t) \equiv \lim _{s \rightarrow 0+} V(t, s) x \text { exists. }
$$

It follows that $w(t) \in \bar{D}$ for each $t$, for by (A.1) and (A.2), the finite product approximates to $W(t, s) x$ all lie in $\bar{D}$. Thus $W(t, s) x \in \bar{D}$, which by (2.4) gives that each $V(t, s) x$, hence, $w(t)$ lies in $\bar{D}$.

Next we argue that $w(t)$ is a strong solution (S.2) to (ACP) $)_{0}$.

(i) For $t_{0}>0$, choose $c \in\left(0, t_{0}\right)$. Then

$$
\begin{aligned}
\lim _{t \rightarrow t_{0}}\left\|w(t)-w\left(t_{0}\right)\right\| & =\lim _{t \rightarrow t_{0}} \lim _{s \rightarrow 0+}\left\|V(t, s) x-V\left(t_{0}, s\right) x\right\| \\
& =\lim _{t \rightarrow t_{0}}\left\|V(t, c) w(c)-V\left(t_{0}, c\right) w(c)\right\|=0,
\end{aligned}
$$

since $w(c) \in \bar{D}$, so that $V(t, c) w(c)$ is a strong solution to $(\mathrm{ACP})_{c}$ with initial value $x=w(c)$.

At $t=0$, we have continuity since by Lemma 2.1,

$$
\begin{aligned}
\lim _{t \rightarrow 0}\|w(t)-x\| & =\lim _{t \rightarrow 0^{+}} \lim _{s \rightarrow 0^{+}}\|V(t, s) x-x\| \\
& \leq \lim _{t \rightarrow 0^{+}} \lim _{s \rightarrow 0^{+}} C \int_{s}^{t}|A(\xi) x| d \xi=0 .
\end{aligned}
$$

(ii) In order to show that $w(t)$ satisfies the differential equation of $(\mathrm{ACP})_{0}$ almost everywhere, it will suffice to show that for each $c>0$,

$$
w^{\prime}(t)+A(t) w(t) \ni 0 \text { for a.e. } t \in(c, T] \text {. }
$$


As argued in (i), $w(t)=V(t, c) w(c)$ is a strong solution to $(\mathrm{ACP})_{c}$ with $x=w(c)$. Hence for a.e. $t \in(c, T]$ :

$$
w^{\prime}(t)=V^{\prime}(t, c) w(c) \ni-A(t) V(t, c) w(c)=-A(t) w(t) .
$$

(iii) $^{\prime}$ To show that $w(t)$ is absolutely continuous on compact subsets of $(0, T)$, let $[a, b] \subseteq(0, T)$ and choose $c \in(0, a)$. Since $V(t, c) w(c)$ is an (S.1) strong solution to $(\mathrm{ACP})_{c}$, the absolute continuity of $w$ on $[a, b]$ follows.

Finally, using the Lipschitz property (2.3), we are able to define $w(t)=\lim _{s \rightarrow 0^{+}} V(t, s) x$ for each $x \in \overline{\mathscr{F}}$, and $w(t)$, as the reader may check, will be a strong solution (S.2) to (ACP) $)_{0}$. As for case (2), it is handled similarly.

Proof of Theorem 2.2. By Theorems 1 and 2 of [3], we may conclude that for every $s>0$, the product integral $W(\cdot, s) x$ exists on $[s, T]$ for all $x \in \bar{D}$.

Now given the relations

$$
\begin{aligned}
& \|W(t, s) x-W(t, s) y\| \leq e^{\omega(t-s)}\|x-y\|, \quad x, y \in \bar{D} . \\
& W(t, r) x=W(t, s) W(s, r) x, \quad r \leq s \leq t, x, y \in \bar{D},
\end{aligned}
$$

the proof of Lemma 2.1 may be used to show

$$
\|W(t, s) x-W(t, r) x\| \leq C e^{\omega(t-s)} \int_{r}^{s}|A(\xi) x| d \xi, \quad x \in \mathscr{F} .
$$

The proofs of (2.5), (2.6) within the context of [5] are straightforward except for one slight difficulty. The product integral $W(t, s) x$ as defined in (1.1) converges in general only along a particular sequence of partitions corresponding to the particular function $h=h_{a}$ used in (C.1) or (C.2). However, as seen by Lemma 4.2 of [3], for purposes of working with a finite collection of functions $h=h_{a}$, we may assume that all product integrals converge along a single sequence of partitions.

The existence of $\tilde{W}(t, 0) x=\lim _{s \rightarrow 0+} W(t, s) x$ for all $x \in \overline{\mathscr{F}}$ thus follows from (2.5) and (2.7). Finally let $u(t)$ be a strong solution (S.1) to $(\mathrm{ACP})_{0}$ with $u(0) \in \mathscr{F}$. Then, by Proposition 2.1, $u(t)=W(t, c) u(c)$ for all $0<c \leq t \leq T$. Hence, using (2.5) we obtain

$$
\begin{aligned}
\|u(t)-\tilde{W}(t, 0) x\| \\
\quad \leq\|W(t, c) u(c)-W(t, c) x\|+\|W(t, c) x-\tilde{W}(t, 0) x\| \\
\quad \leq C e^{\omega(t-s)}\|u(c)-x\|+\|W(t, c) x-\tilde{W}(t, 0) x\|,
\end{aligned}
$$

which approaches zero as $c \rightarrow 0^{+}$. 
3. Examples. We shall have need for Theorem 3.1 below, which can be easily verified by adapting the methods of [2], [3].

THEOREM 3.1. ([2], [3]). Let $X$ be a reflexive Banach space and $A(t)$ be single-valued m-accretive operators with $\operatorname{Dom}(A(t)) \equiv \hat{\mathscr{D}}$. For each $0<a$ $<T$ suppose there exist functions $h_{a}^{i}, i=1, \ldots, m$, such that

(i) each $h_{a}^{i}:[a, T] \rightarrow \mathbf{R}$ is of bounded variation, and

(ii) for a.e.p, $t \in[a, T], 0<\lambda<\lambda_{0}$ and $x \in \hat{\mathscr{D}}$,

$$
\|A(t) x-A(p) x\| \leq \sum_{i=1}^{m}\left|h_{a}^{i}(t)-h_{a}^{i}(p)\right|\|A(p) x\| .
$$

Then $(\mathrm{ACP})_{s}$ has a Lipschitz continuous strong solution (S.1) for each $0<s<T$ and $x \in \hat{\mathscr{D}}$, and this solution lies in $\hat{\mathscr{D}}$.

Our first example is akin to the example presented in [2].

I. Given a bounded region $\Omega$ in $\mathbf{R}^{N}$ having smooth boundary $\partial \Omega$, let $H^{2}(\Omega)$ and $H_{0}^{1}(\Omega)$ be the usual Sobolev spaces. Let $\mu, \nu: \mathbf{R} \rightarrow \mathbf{R}$ be nondecreasing functions having range all of $\mathbf{R}$ and such that $\mu(0)=\nu(0)$ $=0$, and let $\gamma, \phi, \psi:[0, T] \rightarrow \mathbf{R}$ satisfy

(i) $\gamma, \phi, \psi>0$,

(ii) for each $\varepsilon>0$ there exists $\delta>0$ such that $\gamma, \phi, \psi \geq \delta$ on $[\varepsilon, T]$,

(iii) for each $\varepsilon>0$ the functions $\gamma, \phi$, and $\psi$ are of bounded variation on $[\varepsilon, T]$,

(iv) $\gamma, \phi, \psi$ are Lebesgue integrable on $(0, T)$.

Define the operator $\beta(t, u)$ on $L^{2}(\Omega)$ by

$$
\begin{gathered}
\beta(t, u)(x)= \begin{cases}\phi(t) \mu(u(x)), & \text { if } u(x)>0, \\
\psi(t) \nu(u(x)), & \text { if } u(x) \leq 0,\end{cases} \\
\operatorname{Dom}(\beta)=\left\{u \in L^{2}(\Omega): \mu(u), \nu(u) \in L^{2}(\Omega)\right\} .
\end{gathered}
$$

LEMMA 3.1. The operator $\beta(t, u)$ above is $m$-accretive on $L^{2}(\Omega)$. That is, (A.2) and (A.3) are satisfied with $\omega=0$ and $\bar{D}=X=L^{2}(\Omega)$.

Proof. The proof is elementary. We show below that $\beta$ is accretive and leave the remainder of the proof to the reader. Given $u, v \in \operatorname{Dom}(\beta)$, let

$$
\begin{aligned}
& \Omega_{1}=\{x \in \Omega: u(x)>0, v(x)>0\}, \\
& \Omega_{2}=\{x \in \Omega: u(x)>0, v(x) \leq 0\}, \\
& \Omega_{3}=\{x \in \Omega: u(x) \leq 0, v(x)>0\}, \\
& \Omega_{4}=\{x \in \Omega: u(x) \leq 0, v(x) \leq 0\} .
\end{aligned}
$$


Then, for fixed $t$, and all $u, v \in \operatorname{Dom}(\beta)$,

$$
\begin{aligned}
\langle\beta(t, u)- & \beta(t, v), u-v\rangle \\
= & \sum_{i=1}^{4} \int_{\Omega_{t}}[\beta(t, u(x))-\beta(t, v(x))][u(x)-v(x)] d x \\
= & \phi(t) \int_{\Omega_{1}}[\mu(u(x))-\mu(v(x))][u(x)-v(x)] d x \\
& +\psi(t) \int_{\Omega_{4}}[\nu(u(x))-\nu(v(x))][u(x)-v(x)] d x \\
& +\int_{\Omega_{2}}[\phi(t) \mu(u(x))-\psi(t) \nu(v(x))][u(x)-v(x)] d x \\
& +\int_{\Omega_{3}}[\psi(t) \nu(u(x))-\phi(t) \mu(v(x))][u(x)-v(x)] d x .
\end{aligned}
$$

As each of the above integrals in nonnegative, we have $\langle\beta(t, u)-$ $\beta(t, v), u-v\rangle \geq 0$, proving accretiveness.

LEMMA 3.2. For each $t \in[0, T]$, the operator $A(t) u \equiv-\gamma(t) \Delta u+$ $\beta(t, u)$, with $u \in H^{2}(\Omega) \cap H_{0}^{1}(\Omega) \cap \operatorname{Dom}(\beta)$ is m-accretive on $L^{2}(\Omega)$.

Proof. We refer the reader to [1, pp. 80-89], and in particular, to Theorem II.3.6 of this same reference.

THeOREM 3.2. Let $u_{0}(x) \in H^{2}(\Omega) \cap H_{0}^{1}(\Omega) \cap \operatorname{Dom}(\beta)$. Then the initital value problem

$$
\begin{gathered}
\frac{\partial u}{\partial t}-\gamma(t) \Delta u+\beta(t, u)=0 \quad \text { in } \Omega \times[0, T] \\
u(t, x)=0, \quad x \in \partial \Omega, 0 \leq t \leq T \\
u(0, x)=u_{0}(x) \quad \text { in } \Omega
\end{gathered}
$$

has a unique Lipschitz continuous solution $u(t)$ which lies in $H^{2}(\Omega) \cap$ $H_{0}^{1}(\Omega) \cap \operatorname{Dom}(\beta)$ for $0 \leq t \leq T$.

Proof. Given $0<a<T$, let $\delta>0$ be such that $\gamma, \phi, \psi \geq \delta$ on $[a, T]$, and for each $u \in H^{2}(\Omega) \cap H_{0}^{1}(\Omega) \cap \operatorname{Dom}(\beta)$, let $\Omega_{+}=\{x \in \Omega: u(x)>$ $0\}, \Omega_{-}=\{x \in \Omega: u(x) \leq 0\}$. Then, for all $t \in[a, T]$,

$$
\begin{aligned}
\|\beta(t, u)\|^{2} & =\int_{\Omega_{+}} \phi^{2}(t) \mu^{2}(u(x)) d x+\int_{\Omega_{-}} \psi^{2}(t) \nu^{2}(u(x)) d x \\
& \geq \delta^{2}\left(\int_{\Omega_{+}} \mu^{2}(u(x)) d x+\int_{\Omega_{-}} \nu^{2}(u(x)) d x\right) .
\end{aligned}
$$


Also, upon applying Green's Theorem (see e.g. [6, p. 93]) we obtain

$$
\begin{aligned}
\langle\Delta u, \beta(t, u)\rangle= & \int_{\Omega_{+}} \Delta u(x) \beta(t, u(x)) d x \\
& +\int_{\Omega_{-}} \Delta u(x) \beta(t, u(x)) d x \\
= & -\sum_{i=1}^{N}\left[\phi(t) \int_{\Omega_{+}}\left(\frac{\partial u(x)}{\partial x_{i}}\right)^{2} \mu^{\prime}(u(x)) d x\right. \\
& \left.\quad+\psi(t) \int_{\Omega_{-}}\left(\frac{\partial u(x)}{\partial x_{i}}\right)^{2} \nu^{\prime}(u(x)) d x\right]
\end{aligned}
$$

where $\partial u \backslash \partial x_{i}$ are generalized derivatives. Therefore, by (3.1) and (3.2) we have for $t \in[a, T]$,

$$
\begin{gathered}
\|A(t) u\|^{2}=\gamma^{2}(t)\|\Delta u\|^{2}+\|\beta(t, u)\|^{2}-2 \gamma(t)\langle\Delta u, \beta(t, u)\rangle \\
\geq \delta^{2}\left(\|\Delta u\|^{2}+\int_{\Omega_{+}} \mu^{2}(u(x)) d x+\int_{\Omega_{-}} \nu^{2}(u(x)) d x\right) \\
\geq \frac{\delta^{2}}{2}\left(\|\Delta u\|+\left[\int_{\Omega_{+}} \mu^{2}(u(x)) d x+\int_{\Omega_{-}} \nu^{2}(u(x)) d x\right]^{1 / 2}\right)^{2} .
\end{gathered}
$$

Now

$$
\begin{aligned}
\|\beta(t, u)-\beta(p, u)\|^{2}= & \int_{\Omega_{+}}(\phi(t)-\phi(p))^{2} \mu^{2}(u(x)) d x \\
& +\int_{\Omega_{-}}(\psi(t)-\psi(p))^{2} \nu^{2}(u(x)) d x \\
\leq & (|\phi(t)-\phi(p)|+|\psi(t)-\psi(p)|)^{2} \\
& \times\left(\int_{\Omega_{+}} \mu^{2}(u(x)) d x+\int_{\Omega_{-}} \nu^{2}(u(x)) d x\right) .
\end{aligned}
$$

Thus, use of (3.3) and (3.4) yields for all $p, t \in[a, T]$

$$
\begin{array}{r}
\|A(t) u-A(p) u\| \leq(|\gamma(t)-\gamma(p)|+|\phi(t)-\phi(p)|+|\psi(t)-\psi(p)|) \\
\cdot\left(\|\Delta u\|+\left[\int_{\Omega_{+}} \mu^{2}(u(x)) d x+\int_{\Omega_{-}} \nu^{2}(u(x)) d x\right]^{1 / 2}\right) \\
\leq \frac{\sqrt{2}}{\delta}(|\gamma(t)-\gamma(p)|+|\phi(t)-\phi(p)|+|\psi(t)-\psi(p)|)\|A(t) u\| .
\end{array}
$$


The proof now follows upon applying Theorems 2.1 and 3.1.

Consider the special case where $\phi=\psi$ and $\mu(u)=\nu(u)=u^{q}, q$ an odd integer. Then Theorem 3.2 applies to the differential equation

$$
\frac{\partial u}{\partial t}-\gamma(t) \Delta u+\phi(t) u^{q}=0 .
$$

In certain instances (3.5) can alternatively be solved using the method of time scaling. For example, when $\gamma(t)=\phi(t)=1 / \sqrt{t}$, then the change of variable $r=2 \sqrt{t}$ yields solution $u(t, x)=v(2 \sqrt{t}, x)$ to (3.5) where $v(r, x)$ satisfies

$$
\frac{\partial v}{\partial r}-\Delta v+v^{q}=0
$$

See [8] for details and further references. However, when $\gamma, \phi, \psi, \mu$ and $\nu$ are all distinct, then no such scaling techniques would seem to suffice in general.

II. Proposition 3.1. Given an open and bounded region $\Omega \subseteq \mathbf{R}^{N}$, let $X=C(\bar{\Omega})$ and $A$ be a single-valued m-accretive operator on $X$. Suppose $\phi$ : $[0, T] \times \bar{\Omega} \rightarrow \mathbf{R}$ satisfies:

(i) $x \mapsto \phi(t, x)$ is continuous for fixed $t$,

(ii) for each $\varepsilon>0$ there exists $\delta>0$ such that $\delta \leq \phi(t, x)$ for all $(t, x) \in[\varepsilon, T] \times \bar{\Omega}$,

(iii) for all $x \in \bar{\Omega}$ and a.e. $p, t \in[0, T]:|\phi(t, x)-\phi(p, x)| \leq \mid h(t)-$ $h(p) \mid$ where $h$ is a real valued function which is of bounded variation on $[\varepsilon, T]$ for each $\varepsilon>0$, and is Lebesgue integrable on $(0, T)$. Let $A(t) \equiv \phi(t, x) A$ with $\operatorname{Dom}(A(t)) \equiv \operatorname{Dom}(A)$. Then, for each $u_{0} \in$ $\operatorname{Dom}(A)$, the improper product integral $\tilde{W}(t, 0) u_{0}$ of Theorem 2.2 exists.

Proof. The proposition closely follows Proposition 11.1 of [3] where it is seen that the above hypotheses imply that $A(t)$ satisfies (C.2) on each compact subinterval of $(0, T]$. This, along with the integrability of $h$ over $[0, T]$ means that the hypotheses of Theorem 2.2 are satisfied, and the proof follows.

Hence when the initial value problem

$$
\begin{gathered}
\frac{\partial u}{\partial t}+A(t) u=0, \quad 0 \leq t \leq T, \\
u(0)=u_{0} \in \operatorname{Dom}(A)
\end{gathered}
$$

has an (S.1) strong solution $u(t)$, then $u(t)=\tilde{W}(t, 0) u_{0}$. 


\section{Developments in separable Hilbert space.}

TheOREM 4.1. Let $(\mathscr{H},\langle\cdot, \cdot\rangle)$ be a separable Hilbert space with orthonormal basis $\left\{e_{i}\right\}_{i}$. There exists a family of bounded, m-accretive linear operators $\{A(t)\}_{0 \leq t \leq 1}$ on $\mathscr{H}$ with $J_{\lambda}(t) x$ a jointly continuous function of $(\lambda, t, x) \in[0, \infty) \times[0,1] \times \mathscr{H}$ and such that

$$
\int_{0}^{1}\left\langle A(t) e_{i}, e_{i}\right\rangle d t=\infty \text { for all } i
$$

REMARKs. For convenience we may take $\mathscr{H}=l^{2}$ and $\left\{e_{i}\right\}_{i}$ the standard basis (where $e_{i}^{j}$, the $j$ th component of $e_{i}$ is 1 if $j=i$ and otherwise is $0)$. Also, let $\|\cdot\|$ denote the $l^{2}$ norm.

Now viewing the operator $A(t)$ as an infinite dimensional matrix, the theorem asserts that none of the diagonal elements of $A(t)$ are integrable on $[0,1]$. Hence, by the Cauchy inequality,

$$
\int_{0}^{1}\left\|A(t) e_{i}\right\| d t=\infty \quad \text { for all } i
$$

In an arbitrary Banach space when $t \mapsto J_{1}(t)$ is continuous in the operator norm, it is easy to see that $J_{1}^{-1}(t)$, hence $A(t)$ itself, will be operator norm continuous too. It had been hoped that in a separable Hilbert space with $J_{1}(t)$ only strongly continuous and assuming each $A(t)$ is a bounded linear operator, $J_{1}^{-1}(t)$ and hence $A(t)$ would have possessed, if not strong continuity, at least strong or even weak integrability. But, as seen by (4.1), (4.2), this is not the case. Thus, conditions of the sort (P.1) and (P.2) seem to be fundamental for proving existence of solution to $(\mathrm{ACP})_{0}$.

LEMMA 4.1. There exists a unitary matrix $U: l^{2} \rightarrow l^{2}$ which satisfies: for every column index $j$ there is a constant $c_{j}$ such that

$$
U_{i j}=c_{j} / i \text { eventually in } i \text {. }
$$

Proof. We apply the Gram-Schmidt orthonormalization procedure to the column vectors $\left\{v_{1}, e_{2}, e_{3}, \ldots\right\}$ where $v_{1}^{\mathrm{T}}$, the transpose of $v_{1}$, is given by

$$
v_{1}^{\mathrm{T}}=\left(1, \frac{1}{2}, \frac{1}{3}, \ldots\right),
$$

to obtain the orthonormal vectors $\left\{u_{1}, u_{2}, \ldots\right\}$. Thus $u_{1}=(\sqrt{6} / \pi) v_{1}$ and given $u_{1}, u_{2}, \ldots, u_{i-1}$, we have

$$
u_{i}=\left\|e_{i}-\sum_{j=1}^{i-1}\left\langle e_{i}, u_{j}\right\rangle u_{j}\right\|^{-1}\left(e_{i}-\sum_{j=1}^{i-1}\left\langle e_{i}, u_{j}\right\rangle u_{j}\right) .
$$


Let $U$ be the matrix having column vectors $u_{1}, u_{2}, \ldots, u_{l}, \ldots$ Then clearly $U^{\mathrm{T}} U=I$. As well, it can be shown that $U U^{\mathrm{T}}=I$. Hence $U$ is unitary.

Obviously (4.3) holds for index $j=1$. Suppose it holds for indices $j=1,2, \ldots, k-1$. Then, by (4.4) there is a constant $\tilde{c}_{k}$ such that

$$
\begin{aligned}
U_{\imath k} & =\left\langle e_{i}, u_{k}\right\rangle=\tilde{c}_{k}\left(e_{k}^{i}-\sum_{j=1}^{k-1}\left\langle e_{k}, u_{J}\right\rangle\left\langle e_{i}, u_{J}\right\rangle\right) \\
& =\left(-\tilde{c}_{k} \sum_{j=1}^{k-1}\left\langle e_{k}, u_{j}\right\rangle c_{J}\right) / i \quad \text { eventually in } i .
\end{aligned}
$$

Hence, by induction, the proof is complete.

Proof of Theorem 4.1. For each $i$ let $\gamma_{l}(t)$ be a continuous function which satisfies $0<\gamma_{i}(t) \leq 1$ and

$$
\gamma_{i}(t)= \begin{cases}1 / 3^{i} & t \in\left[(5 / 4) / 2^{i+1},(4 / 5) / 2^{\imath}\right], \\ 1, & t \notin\left[1 / 2^{i+1}, 1 / 2^{\imath}\right] .\end{cases}
$$

Let $\Gamma(t)$ be the diagonal operator having diagonal elements

$$
\Gamma_{i i}(t)=\gamma_{i}(t), \quad i=1,2, \ldots
$$

Define

$$
K(t)=U^{\mathrm{T}} \Gamma(t) U
$$

where $U$ is the unitary operator of Lemma 4.1. Therefore, for every index $j$ there is a constant $c_{j}$ and an index $I(j)$ such that

$$
U_{l j}=c_{j} / i, \text { for all } i \geq I(j) .
$$

Now $K^{-1}(t)=U^{\mathrm{T}} \Gamma^{-1}(t) U$ which yields

$$
\begin{aligned}
\int_{0}^{1}\left\langle K^{-1}(t) e_{j}, e_{j}\right\rangle d t & =\int_{0}^{1} \sum_{i=1}^{\infty} \frac{U_{l j}^{2}}{\gamma_{l}(t)} d t \geq \sum_{l=I(J)}^{\infty} \int_{1.2 / 2^{1+1}}^{.8 / 2^{i}} \frac{U_{l j}^{2}}{\gamma_{l}(t)} d t \\
& =.2 \sum_{i=I(j)}^{\infty} \frac{c_{J}^{2}}{i^{2}} \frac{3^{l}}{2^{i}}=\infty .
\end{aligned}
$$

Thus, if $A(t)=K^{-1}(t)-I, 0 \leq t \leq 1$, then $A(t)$ is a family of bounded, linear operators which satisfies (4.1). Furthermore, each $A(t)$ is $m$-accretive since $[I+A(t)]^{-1}=K(t)$ has domain all of $l^{2}$ and for every $x \in l^{2}$ :

$$
\begin{aligned}
\langle A(t) x, x\rangle & =\left\langle\Gamma^{-1}(t) U x, U x\right\rangle-\langle x, x\rangle=\left\langle\Gamma^{-1}(t) y, y\right\rangle-\langle y, y\rangle \\
& =\sum_{i=1}^{\infty}\left\langle e_{i}, y\right\rangle^{2}\left(\gamma_{i}^{-1}(t)-1\right) \geq 0,
\end{aligned}
$$

where $y=U x$. 
Finally, we verify continuity of $(\lambda, t, x) \rightarrow J_{\lambda}(t) x$. For $0<t \leq 1$, this follows readily from $m$-accretiveness and continuity of $A(t)$. For $t=0$, let $\left\{t_{m}\right\}_{m}$ be any sequence which approaches zero and such that $t_{m} \in\left[2^{-(m+1)}, 2^{-m}\right], m=1,2, \ldots$ Then for $x, y \in l^{2}$ and $\lambda, \mu \geq 0$ we have $J_{\lambda}(0) x=x$ and

$$
\begin{aligned}
\| J_{\lambda}(0) x-J_{\mu}\left(t_{m}\right) & y\|\leq\| x-y\|+\| J_{\mu}\left(t_{m}\right) y-y \| \\
& =\|x-y\|+\left\|U^{\mathrm{T}}\left\{\left[(1-\mu) I+\mu \Gamma^{-1}\left(t_{m}\right)\right]^{-1} U y-U y\right\}\right\| \\
& =\|x-y\|+\left|1-\left(1-\mu+\mu \gamma_{m}^{-1}\left(t_{m}\right)\right)^{-1}\right| \sqrt{\left\langle e_{m}, z\right\rangle} \\
& \leq\|x-y\|+\sqrt{\left\langle e_{m}, z\right\rangle},
\end{aligned}
$$

where $z=U y$. Thus $J_{\mu}\left(t_{m}\right) y$ approaches $J_{\lambda}(0) x$ as $\left(\mu, t_{m}, y\right)$ approaches $(\lambda, 0, x)$.

\section{REFERENCES}

[1] V. Barbu, Nonlinear Semigroups and Differential Equations in Banach Spaces, Noordhoff Internat. Publ., (1976).

[2] M. G. Crandall and A. Pazy, Nonlinear evolution equations in Banach spaces, Israel J. Math., 11 (1972), 57-94.

[3] L. C. Evans, Nonlinear evolution equations in an arbitrary Banach space, Israel J. Math., 26 (1977), 1-42.

[4] M. A. Freedman, Product integrals of continuous resolvents: existence and nonexistence, Israel J. Math., 46 (1983), 145-160.

[5] _ Riemann step function approximation of Bochner integrable functions, to appear in Proc. Amer. Math. Soc.

[6] S. Fučik and Kufner, Nonlinear Differential Equations, Elsevier, New York (1980).

[7] A. Pazy, Semigroups of Linear Operators and Applications to Partial Differential Equations, Springer-Verlag, New York (1983).

[8] R. E. Showalter, Singular nonlinear evolution equations, Rocky Mountain J. Math., 10 (1980), 499-507.

Received April 12, 1984.

VANDERBILT UNIVERSITY

NASHVILLE, TN 37235 


\section{PACIFIC JOURNAL OF MATHEMATICS EDITORS}

\author{
V. S. VARADARAJAN (Managing Editor) \\ University of California \\ Los Angeles, CA 90024 \\ Charles R. DePrima \\ California Institute of Technology \\ Pasadena, CA 91125 \\ R. FINN \\ Stanford University \\ Stanford, CA 94305
}

\author{
HeRmanN FlaschKa \\ University of Arizona \\ Tucson, AZ 857.21
}

RAMESH A. GANGOLli

University of Washington

Seattle, WA 98195

ROBION KIRBY

University of California

Berkeley, CA 94720
C. C. MOORE

University of California

Berkeley, CA 94720

H. SAMELSON

Stanford University

Stanford, CA 94305

HAROLD STARK

University of California, San Diego

La Jolla, CA 92093

\section{ASSOCIATE EDITORS}
R. ARENS
E. F. BECKENBACH
B. H. NeumanN
F. WOLF
K. YoSHIDA
(1906-1982)

\section{SUPPORTING INSTITUTIONS}

\begin{abstract}
UNIVERSITY OF ARIZONA
UNIVERSITY OF BRITISH COLUMBIA

CALIFORNIA INSTITUTE OF TECHNOLOGY

UNIVERSITY OF CALIFORNIA

MONTANA STATE UNIVERSITY

UNIVERSITY OF NEVADA, RENO

NEW MEXICO STATE UNIVERSITY

OREGON STATE UNIVERSITY
\end{abstract}

\author{
UNIVERSITY OF OREGON \\ UNIVERSITY OF SOUTHERN CALIFORNIA \\ STANFORD UNIVERSITY \\ UNIVERSITY OF HAWAII \\ UNIVERSITY OF TOKYO \\ UNIVERSITY OF UTAH \\ WASHINGTON STATE UNIVERSITY \\ UNIVERSITY OF WASHINGTON
}

The Supporting Institutions listed above contribute to the cost of publication of this Journal, but they are not owners or publishers and have no responsibility for its content or policies.

Mathematical papers intended for publication in the Pacific Journal of Mathematics should be in typed form or offset-reproduced (not dittoed), double spaced with large margins. Please do not use built up fractions in the text of the manuscript. However, you may use them in the displayed equations. Underline Greek letters in red, German in green, and script in blue. The first paragraph must be capable of being used separately as a synopsis of the entire paper. In particular it should contain no bibliographic references. Please propose a heading for the odd numbered pages of less than 35 characters. Manuscripts, in triplicate, may be sent to any one of the editors. Please classify according to the scheme of Math. Reviews, Index to Vol. 39. Supply name and address of author to whom proofs should be sent. All other communications should be addressed to the managing editor, or Elaine Barth, University of California, Los Angeles, California 90024.

There are page-charges associated with articles appearing in the Pacific Journal of Mathematics. These charges are expected to be paid by the author's University, Government Agency or Company. If the author or authors do not have access to such Institutional support these charges are waived. Single authors will receive 50 free reprints; joint authors will receive a total of 100 free reprints. Additional copies may be obtained at cost in multiples of 50 .

The Pacific Journal of Mathematics is issued monthly as of January 1966. Regular subscription rate: $\$ 190.00$ a year (5 Vols., 10 issues). Special rate: $\$ 66.00$ a year to individual members of supporting institutions.

Subscriptions, orders for numbers issued in the last three calendar years, and changes of address should be sent to Pacific Journal of Mathematics, P.O. Box 969, Carmel Valley, CA 93924, U.S.A. Old back numbers obtainable from Kraus Periodicals Co., Route 100, Millwood, NY 10546.

The Pacific Journal of Mathematics at P.O. Box 969, Carmel Valley, CA 93924 (ISSN 0030-8730) publishes 5 volumes per year. Application to mail at Second-class postage rates is pending at Carmel Valley, California, and additional mailing offices. Postmaster: Send address changes to Pacific Journal of Mathematics, P.O. Box 969, Carmel Valley, CA 93924.

PUBLISHED BY PACIFIC JOURNAL OF MATHEMATICS, A NON-PROFIT CORPORATION

Copyright $\odot 1985$ by Pacific Journal of Mathematics 


\section{Pacific Journal of Mathematics}

Vol. 120, No. $2 \quad$ October, 1985

Philip Marshall Anselone and Mike Treuden, Regular operator

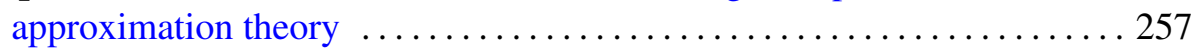

Giuseppe Baccella, Semiprime $\aleph-Q F 3$ rings $\ldots \ldots \ldots \ldots \ldots \ldots \ldots \ldots . \ldots \ldots$

Earl Robert Berkson and Thomas Alastair Gillespie, The generalized M.

Riesz theorem and transference $\ldots \ldots \ldots \ldots \ldots \ldots \ldots \ldots . \ldots 279$

Joachim Boidol, A Galois-correspondence for general locally compact

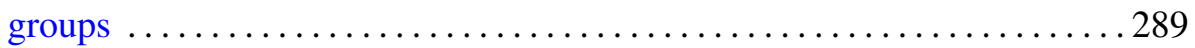

Joseph Eugene D'Atri, Josef Dorfmeister and Yan Da Zhao, The isotropy

representation for homogeneous Siegel domains ............... 295

C. Debiève, On Banach spaces having a Radon-Nikodým dual

Michael Aaron Freedman, Existence of strong solutions to singular

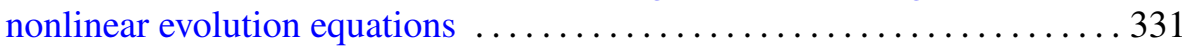

Francisco Jose Freniche, Grothendieck locally convex spaces of continuous

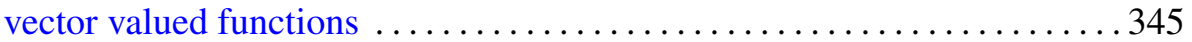

Hans-Peter Künzi and Peter Fletcher, Extension properties induced by complete quasi-uniformities . ............................ 357

Takaŝi Kusano, Charles Andrew Swanson and Hiroyuki Usami, Pairs of

positive solutions of quasilinear elliptic equations in exterior domains . . 385

Angel Rafael Larotonda and Ignacio Zalduendo, Spectral sets as Banach

manifolds

J. Martínez-Maurica and C. Pérez García, A new approach to the

Kreı̆n-Milman theorem

Christian Pommerenke, On the boundary continuity of conformal maps . . . 423

M. V. Subba Rao, Some Rogers-Ramanujan type partition theorems

Stephen Edwin Wilson, Bicontactual regular maps .........

Jaap C. S. P. van der Woude, Characterizations of (H)PI extensions

Kichoon Yang, Deformation of submanifolds of real projective space

Subhashis Nag, Errata: "On the holomorphy of maps from a complex to a

real manifold" 